[Article]

\title{
核壳结构碳化铇/碳化铇铁复合材料的制备与电催化活性
}

\author{
陈 辉 ${ }^{1}$ 陈 丹 ${ }^{1}$ 谢伟沝 ${ }^{1}$ 郑 翔 ${ }^{1}$ 李国华 ${ }^{1,2,3, *}$ \\ ('浙江工业大学化学工程学院, 杭州 310032; 2绿色合成技术国家重点实验室培育基地, 杭州 310032; \\ 3 浙江工业大学纳米科学与工程技术研究中心, 杭州 310032)
}

\begin{abstract}
摘要: 以铁黄为载体, 偏铇酸铵为铇源, 将直接包覆与原位还原碳化技术相结合制备了碳化铇/碳化铇铁复合 材料. 经 X射线衍射 $(X R D)$ 分析和透射电子显微镜(TEM)观察, 复合材料的主要物相为碳化铇铁 $\left(\mathrm{Fe}_{3} \mathrm{~W}_{3} \mathrm{C}\right.$ )、碳化 铇 $(W C)$ 和碳化二铇 $\left(W_{2} C\right)$, 且构成了以 $F_{3} W_{3} C$ 为核、 $W C$ 和 $W_{2} C$ 为壳的核壳结构. 采用三电极体系循环伏安法 测试了复合材料在酸性、中性和碱性体系中对甲醇的电催化氧化活性. 结果表明, 与颗粒状碳化铇和介孔空心 球状碳化铇相比, 复合材料的电催化活性有了明显的提高; 进一步研究发现, 复合材料的电催化活性不仅受到 体系性质的影响, 还与其物相组成和微结构相关. 上述结果说明, 通过控制复合材料的物相组成及微结构, 以 及反应体系的性质可实现对其电催化活性的调控; 同时表明, 核壳结构是提高碳化铇催化材料活性的有效途径 之一。
\end{abstract}

关键词：碳化铇；碳化铇铁; 核壳结构; 电催化活性; 调控

中图分类号: 0646

\section{Preparation and Electrocatalytic Activity of Tungsten Carbide and Tungsten-Iron Carbide Composite with Core-Shell Structure}

\author{
CHEN Hui $\quad$ CHEN Dan ${ }^{1} \quad$ XIE Wei-Miao ${ }^{1} \quad$ ZHENG Xiang ${ }^{1} \quad$ LI Guo-Hua ${ }^{1,2,3, *}$ \\ ('School of Chemical Engineering, Zhejiang University of Technology, Hangzhou 310032, P. R. China; ${ }^{2}$ State Key Breeding Base \\ of Green Chemistry Synthesis Technology, Hangzhou 310032, P. R. China; ${ }^{3}$ Research Center of Nanoscience and Engineering \\ Technology, Zhejiang University of Technology, Hangzhou 310032, P. R. China)
}

\begin{abstract}
A tungsten carbide and tungsten-iron carbide composite with a core-shell structure was prepared through a combination of surface coating and in situ reduction-carbonization, using ammonium metatungstate as the tungsten source and iron hydroxide as the iron source. The main crystal phases of the composite were tungsten-iron carbide $\left(\mathrm{Fe}_{3} \mathrm{~W}_{3} \mathrm{C}\right)$, monotungsten carbide $(\mathrm{WC})$, and bitungsten carbide $\left(\mathrm{W}_{2} \mathrm{C}\right)$. In the core-shell composite, $\mathrm{Fe}_{3} \mathrm{~W}_{3} \mathrm{C}$ formed the core, and the shell consisted of $\mathrm{WC}$ and $\mathrm{W}_{2} \mathrm{C}$. The electrocatalytic activity for methanol oxidation of the composite was measured by cyclic voltammetry with a three-electrode system in acidic, neutral, and alkaline aqueous solutions. The results show that the electrocatalytic activity of the composite is higher than those of tungsten carbide particles and mesoporous hollow microspheres. The activity is affected by the properties of the solution in which the reaction is performed, and is related to the crystal phase and microstructure of the composite. These results indicate that the electrocatalytic activity of tungsten carbide can be adjusted by changing the properties of the reaction solution and controlled by adjusting the crystal phase and microstructure of the composite. Furthermore, the activity can be improved through formation of a core-shell structure; this is an efficient way to improve the electrocatalytic activity of tungsten carbide.
\end{abstract}

Received: November 7, 2013; Revised: February 24, 2014; Published on Web: February 24, 2014.

"Corresponding author. Email: nanozjut@zjut.edu.cn; Tel: +86-571-88320418.

The project was supported by the National Natural Science Foundation of China $(21173193,21301154)$ and Science and Technology Project of Zhejiang Province, China (2013C37104, 2009R50002-15).

国家自然科学基金(21173193, 21301154)及浙江省科技厅科技项目(2013C37104, 2009R50002-15)资助

(c) Editorial office of Acta Physico-Chimica Sinica 
Key Words: Tungsten carbide; Tungsten-iron carbide; Core-shell structure; Electrocatalytic activity; Adjusting

\section{1 引言}

碳化铇因具有类铂催化性能,, 2 可用作化学催 化和电催化领域的催化剂, ${ }^{3-5}$ 且在催化反应过程中 不易被 $\mathrm{CO} 、 \mathrm{H}_{2} \mathrm{~S}$ 等气体中毒 ${ }^{6}$ 而受到了广泛关注. 碳 化铇的上述特性使其具备了替代或部分替代铂等 贵金属催化剂的潜质. 现有研究表明, 碳化铇的催 化活性远不及铂等贵金属. 7.8 因此,如何提高碳化铇 催化活性, 并使其接近铂等贵金属催化剂是碳化铇 替代和部分替代铂等贵金属催化剂的关键, 也是碳 化铇基催化材料研究的热点和走向工业应用的核 心问题之一.

金属化的表面结构是碳化铇具有类铂催化性 能的原因所在, ${ }^{9}$ 若能够调控碳化铇的表面电子结 构, 必可调控碳化铇的催化活性. 基于这样的认识, 国内外专家学者开展了不少有针对性的研究工 作, ${ }^{10-19}$ 比如, 采用模板法将碳化铇制备成具有一定 结构和形貌催化材料, ${ }^{10,11}$ 应用原位还原碳化和碳包 覆将碳化铇制备成纳米材料, ${ }^{12-18}$ 将碳化铇与其它材 料复合, ${ }^{19}$ 这些均不同程度地提高了碳化铇的催化 活性. 本课题组在这方面也开展了一系列研究工 作. 首先, 利用碳纳米管优越的电子性能、管状结构 和比表面积大等特点, 将偏铇酸铵负载于硝化后的 碳纳米管上, 经还原碳化后获得了碳化铇与碳纳米 管复合材料: ${ }^{20}$ 其次, 以具有三维联通孔道结构的天 然沸石为载体制备了碳化铇与天然沸石纳米复合 材料, ${ }^{21}$ 再次, 利用 $\mathrm{Ti}^{4+}$ 的空 $d$ 轨道的独特性质, ${ }^{22}$ 以金 红石型纳米二氧化钛为载体制备了碳化铇与金红 石纳米复合材料, ${ }^{23}$ 最后, 以铁黄为载体, 偏铇酸铵 为铇源, 将表面包覆法与原位还原碳化技术相结 合, 制备出了具有核壳结构的碳化铇与碳化二铇纳 米复合材料. ${ }^{24}$ 上述复合材料均在一定程度上提高 了碳化铇的电催化活性, 但也面临一些问题. 碳纳 米管虽具有优越的电子性能和管状结构, 但在电化 学环境下不稳定会被氧化, 从而导致复合材料的结 构与性能发生变化. 以天然沸石和二氧化钛为载体 虽然可克服上述问题, 但其电催化活性和导电性较 差, 且复合材料中碳化铇分布的均匀性也较难控制.

为寻找解决上述问题的技术方法, 本文提出制 备碳化铇铁 $\left(\mathrm{Fe}_{3} \mathrm{~W}_{3} \mathrm{C}\right)$ 与碳化铇 (WC) 核壳结构复合 材料的新思路. 这是因为, 一方面, $\mathrm{Fe}_{3} \mathrm{~W}_{3} \mathrm{C}$ 和 $\mathrm{WC}$ 均
对甲醇的电催化氧化具有较好的催化活性: ${ }^{25}$ 另一 方面, $\mathrm{Fe}_{3} \mathrm{~W}_{3} \mathrm{C}$ 具有良好的导电性; 更为重要的是核 壳结构复合材料可利用核和壳层的特点对材料的 性能进行调控, 可明显提升复合材料的催化性能. ${ }^{26}$ 基于上述因素, 本文利用铁黄比表面积大和吸附性 能优越等特点, 采用包覆技术将偏铇酸铵均匀地吸 附于其外表面, 并利用铁黄中的铁元素在还原碳化 过程中能够掺入碳化铇内形成 $\mathrm{Fe}_{3} \mathrm{~W}_{3} \mathrm{C}$, 以增强复合 材料导电性的特点, 将表面包覆和原位还原碳化技 术相结合, 制备出具有核壳结构的 $\mathrm{Fe}_{3} \mathrm{~W}_{3} \mathrm{C}$ 与 $\mathrm{WC}$ 复 合材料, 并首次报道了 $\mathrm{Fe}_{3} \mathrm{~W}_{3} \mathrm{C}$ 与 $\mathrm{WC}$ 核壳结构复合 材料对甲醇的电催化性能.

\section{2 实验部分}

\section{1 样品制备}

前驱体制备. 称取一定量的偏铇酸铵(AMT, 分 析纯, 湖南株洲硬质合金厂), 溶解于 $400 \mathrm{~mL}$ 去离子 水中, 再按比例称取相应质量的铁黄 $(\mathrm{FeOOH}$, 分析 纯, 浙江升华集团德清华源颜料有限公司)加入其 中, 在恒温磁力搅拌器内 $60^{\circ} \mathrm{C}$ 条件下持续摚拌 $3 \mathrm{~h}$, 静置, 去上层清液后于烘箱内 $80^{\circ} \mathrm{C}$ 干燥 $10 \mathrm{~h}$, 研磨 即得前驱体.

样品制备. 将前驱体装于石英舟内, 再置于恒 温管式炉中, 以 $\mathrm{H}_{2}$ 为还原气体、 $\mathrm{CH}_{4}$ 为碳源, 在 $900{ }^{\circ} \mathrm{C}$ 条件下还原碳化 $4 \mathrm{~h}$, 冷却后即得碳化铇铁与碳化铇 复合材料.

系列样品制备. 按上述方法分别制备 AMT与 $\mathrm{FeOOH}$ 质量比 5、10、15 和 20 的四个样品, 并分别标 注为样品 $1 、 2 、 3$ 和 4 .

\section{2 样品表征}

样品物相, 即 X射线衍射分析(XRD)分析采用 荷兰 PANalytical 公司生产的 X'Pert PRO 型 X 射线 衍射仪, 测试时采用 $\mathrm{Cu} K_{a}$ 射线源 $(\lambda=0.1541 \mathrm{~nm})$, 工 作电压为 $40 \mathrm{kV}$, 电流为 $40 \mathrm{~mA}$, 扫描范围 $5^{\circ}-80^{\circ}$, 记 数步长 $0.033^{\circ}$. XRD 数据用仪器自带分析软件 High Score进行处理, 处理过程中仪器本征宽化采用厂家 提供的多晶硅标准样品进行校正与扣除; 样品形貌 采用场发射扫描电子显微镜(SEM Hitachi S-4700II) 进行观察; 样品微结构采用高分辨透射电子显微镜 (TEM Tecnai G2 F30)进行表征; 样品的化学组成采 


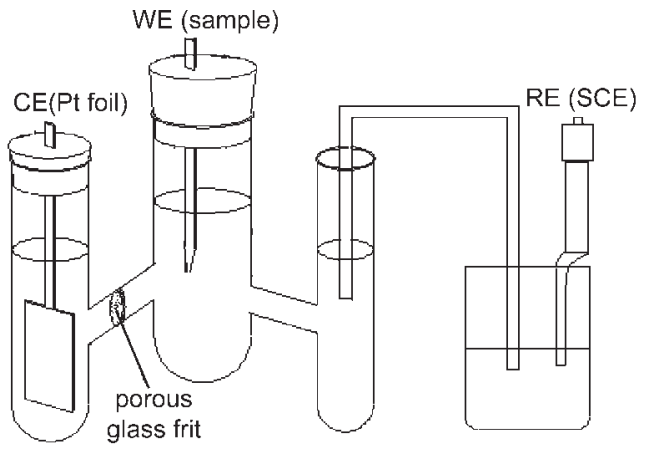

图 1 三电极体系示意图

Fig.1 Schematic diagram of tri-electrode system WE: working electrode; RE: reference electrode; $\mathrm{CE}$ : counter electrode 用 X射线能量散射谱(EDS)进行分析.

\section{3 电化学性能测试}

样品的电催化性能用 CHI620B 电化学工作站 测试. 测试过程中采用三电极体系(如图 1 所示)、测 试时用循环伏安法 $(\mathrm{CV})$, 工作电极(WE) 为粉末微电 极, ${ }^{27}$ 参比电极 $(\mathrm{RE})$ 为饱和甘录电极 (SCE), 辅助电极 (CE) 为光亮铂电极, 盐桥由氯化钾与琼脂质量比为 10:1 的混合物制备而成. 测试前为了形成稳定的三 相反应界面, 将电极在电解液中浸泡 $3 \mathrm{~h}$. 测试过程 中溶液电阻采用正反馈技术进行欧姆补偿.

\section{3 样品表征}

\section{1 前驱体样品表征}

\subsubsection{XRD表征}

图 2 为前驱体的 $\mathrm{XRD}$ 结果. 从图 2 中可以看到, 前驱体的 XRD 图中有十多处衍射峰, 其中, $2 \theta$ 为 $8.5560^{\circ} 、 9.9328^{\circ} 、 27.8638^{\circ}$ 等处的衍射峰可归属为铇 酸铵 (PDF No. 070-2271), $2 \theta$ 为 $21.2159^{\circ} 、 33.2233^{\circ}$ 、 $36.6426^{\circ}$ 等处的衍射峰可归属为 $\mathrm{FeOOH}$ (PDF No. 081-0463), 即前驱体主要含有两种物相: 铇酸铵

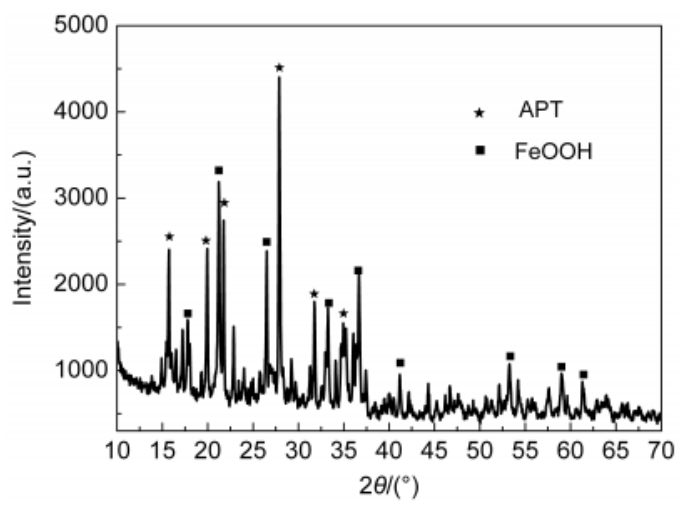

图 2 前驱体的 XRD 图谱

Fig.2 X-ray diffraction (XRD) pattern of the precursor APT: ammonium tungstate
(APT) 和 $\mathrm{FeOOH}$. 这说明, 将偏铇酸铵溶于水并负载 于铁黄表面之后, 铁黄的物相基本没有改变, 偏铇酸 铵转变成了钨酸铵.

\subsubsection{SEM表征}

图 3 是前驱体的扫描电子显微镜(SEM)照片, 其 中, 图 3A 为前驱体的形貌, 图 3B 为前驱体的表面结 构. 从图 3B 中可看出, 前驱体大多呈短小柱状, 长短 不一, 柱体的平均长度在 $500 \mathrm{~nm}$ 左右, 宽 80-150 $\mathrm{nm}$, 柱体表面比较光滑. 这与铁黄载体的形貌 ${ }^{24}$ 有一 定的差异. 这是由于在前驱体制备过程中, 经过摚 拌、加热、干燥以及研磨后, 载体铁黄的形貌有一定 程度的破坏, 负载后的前驱体在干燥和研磨过程中 也会断裂.

\subsubsection{TEM表征}

图 4 为前驱体的透射电镜 $(\mathrm{TEM})$ 照片. ${ }^{24}$ 从图 $4 \mathrm{~A}$ 中可看出, 前驱体呈棒状, 保持了铁黄的形貌, 长度 多在 $500 \mathrm{~nm}$ 以上, 粗细不等, 直径 50-70 nm; 与铁黄 (见图 4A 中嵌入图)相比, 前驱体中间灰度较高, 边缘 灰度较低, 两者之间有明显的界线. 这反映前驱体可 分为载体和负载层两个部分, 后者厚度 6.5-9.0 nm, 如图 4A 所示. 图 4B 为的图 4A 的局部放大, 经测量, 负载层的晶面间距为 $0.7858 \mathrm{~nm}$, 与 APT (PDF No. 070-2271)的(111)晶面间距接近. 这与 XRD 的分析结 果一致. 依据上述结果可认为, 前驱体是由 APT 和
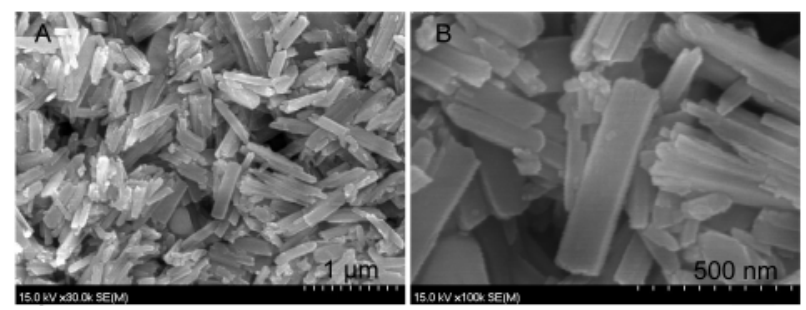

图 3 前驱体的 SEM 照片

Fig.3 Scanning electron microscope (SEM) images of the precursor

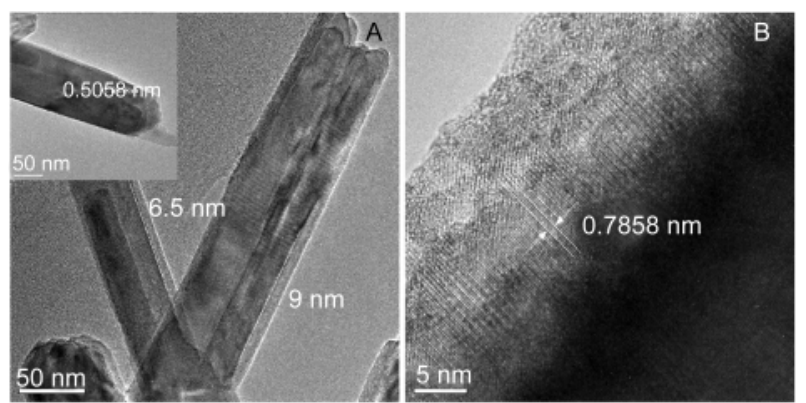

图 4 前驱体的透射电子显微镜照片 ${ }^{24}$

Fig.4 Transmission electron microscope (TEM) images of precursor $^{24}$ 


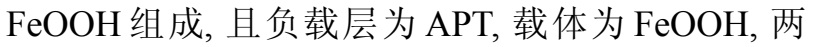
者构成了具有典型核壳结构的棒状前驱体.

\section{2 样品表征}

\subsubsection{XRD 表征}

图 5 是以偏铇酸铵和铁黄为原料, 按不同 AMT 与 $\mathrm{FeOOH}$ 比例制备的前驱体经还原碳化后样品的 $\mathrm{XRD}$ 分析结果. 从图 5 中可看到, 随着制备过程中 $\mathrm{AMT}$ 与 $\mathrm{FeOOH}$ 比例的变化, 样品的主要衍射峰没 有明显变化, 其中, $2 \theta$ 为 $13.79^{\circ} 、 22.64^{\circ} 、 32.25^{\circ}$ 、 $35.24^{\circ} 、 39.78^{\circ} 、 42.31^{\circ} 、 46.26^{\circ} 、 59.48^{\circ} 、 64.49^{\circ} 、$ $69.30^{\circ} 、 72.23^{\circ}$ 和 $73.99^{\circ}$ 处的衍射峰可归属于碳化铇 铁 $\mathrm{Fe}_{3} \mathrm{~W}_{3} \mathrm{C}$ (PDF No. 94-004-3230), 且 $2 \theta$ 为 $42.31^{\circ}$ 、 $39.78^{\circ} 、 32.25^{\circ} 、 35.24^{\circ}$ 和 $46.26^{\circ}$ 处的衍射峰是碳化铇 铁的特征峰; $2 \theta$ 为 $35.56^{\circ}$ 处的衍射峰可归属于碳化 铇 WC(PDF No. 051-0939) 的特征峰, 即最强峰; $2 \theta$ 为 $38.13^{\circ}$ 处的衍射峰可归属于碳化二铇 $\mathrm{W}_{2} \mathrm{C}(\mathrm{PDF}$ No. 089-2731)的特征峰. 这说明四个样品的主要物 相均为 $\mathrm{Fe}_{3} \mathrm{~W}_{3} \mathrm{C} 、 \mathrm{WC}$ 和 $\mathrm{W}_{2} \mathrm{C}$. 其中, 后两种物相只看 到了最强衍峰, 其它衍射峰未能看到的原因是它们 被碳化铇铁的衍射峰掩盖所致.

比较上述四个样品 $\mathrm{WC}$ 和 $\mathrm{W}_{2} \mathrm{C}$ 的衍射峰强可 知, 样品 1 中 $\mathrm{WC}$ 的特征峰衍射强度较弱, $\mathrm{W}_{2} \mathrm{C}$ 特征 峰衍射强度较强; 与样品 1 相比, 样品 2 中 $\mathrm{WC}$ 特征 峰的衍射强度明显增强, $\mathrm{W}_{2} \mathrm{C}$ 特征峰的衍射强度却 有所下降; 与样品 2 相比, 样品 3 中 $\mathrm{W}_{2} \mathrm{C}$ 特征峰的衍 射强度没有较大改变, 但 WC 特征峰的衍射强度增 强; 样品 4 中 $\mathrm{WC}$ 和 $\mathrm{W}_{2} \mathrm{C}$ 特征峰的衍射强度均较弱. 上述结果说明, $\mathrm{W}_{2} \mathrm{C}$ 特征峰的衍射强度随着制备过 程中 $\mathrm{AMT}$ 与 $\mathrm{FeOOH}$ 比例的增加而持续减弱; $\mathrm{WC}$ 特征峰的衍射强度在 AMT 与 $\mathrm{FeOOH}$ 比例为 5-15

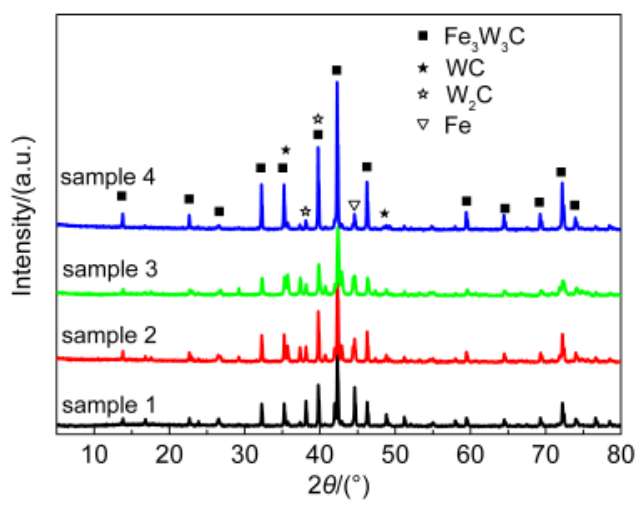

图 $5 \mathrm{AMT}$ 与 $\mathrm{FeOOH}$ 不同质量比样品的 XRD 图谱

Fig.5 XRD patterns of the samples prepared with different mass ratios of AMT to $\mathrm{FeOOH}$

$m$ (AMT): $m(\mathrm{FeOOH})$ : sample 1, 5; sample 2, 10; sample 3, 15; sample 4, 20. AMT: metatungstate
范围内持续增强, 当这一比例为 20 时却明显减弱. 对于同一物相而言, 衍射峰强度与物质的量有着一 定的相关性. 据此, 上述结果说明在样品制备过程 中, 当 $\mathrm{AMT}$ 与 $\mathrm{FeOOH}$ 的比例在 5-20范围内时, 随 着比例的增大, $\mathrm{W}_{2} \mathrm{C}$ 含量逐渐降低; 当比例在 5-15 范围内时, $\mathrm{WC}$ 含量随着比例的增大而逐渐增加, 当 比例增大到 20 时, $\mathrm{WC}$ 含量下降.

比较样品中 $\mathrm{F}_{3} \mathrm{~W}_{3} \mathrm{C}$ 的衍射峰强度可知, 样品 4 的衍射峰强度最高, 其次是样品 2 , 再是样品 3 , 最弱 的是样品 1. 这说明样品 4 中 $\mathrm{F}_{3} \mathrm{~W}_{3} \mathrm{C}$ 的含量最多, 其 次是样品 2 , 再是样品 3 , 含 $\mathrm{F}_{3} \mathrm{~W}_{3} \mathrm{C}$ 最少的是样品 1 .

\subsubsection{SEM}

图 6 为样品 2 的扫描电子显微镜照片. 从图 6 中 可看出, 样品的颗粒为不规则粒状, 粒径介于 150$500 \mathrm{~nm}$ 之间, 颗粒粒径较为均匀, 分散相对较好. 这 与其前驱体的棒状形貌有明显差别. 这是因为前驱 体在高温还原碳化过程中发生了融熔和重结晶, 从 而导致样品颗粒的形貌由棒状转变成了颗粒状.

\subsubsection{TEM}

图 7 为样品的 TEM 照片, 其中 $A 、 B 、 C$ 和 $D$ 分别 为样品 $1 、 2 、 3$ 和 4 的整体形貌. 从图 7 中可以看到, 样品 $1 、 2$ 和 3 的颗粒粒径均在 200-500 nm 范围内, 样品 4 的颗粒粒径下降到 $200 \mathrm{~nm}$ 以下, 即随着制备 过程中 $\mathrm{AMT}$ 与 $\mathrm{FeOOH}$ 比例的增加样品的颗粒粒径 逐渐减小. 图 7 中的四个嵌入图均为各自样品颗粒 的局部放大. 从图 7 中可看出, 样品 1 中颗粒的核壳 结构不是很清晰, 如图 7A 中嵌入图所示, 其余 3 个 样品颗粒的核壳结构清晰可见, 壳层的厚度均在 $100 \mathrm{~nm}$ 以下, 如图 7B、7C 和 7D 中嵌入图所示. 比较 核壳结构的壳层厚度和均匀性, 由样品 2 到样品 4 , 壳层的厚度逐渐减小, 均匀性逐渐增加. 这说明样 品的微结构和 AMT 与 $\mathrm{FeOOH}$ 比例相关.

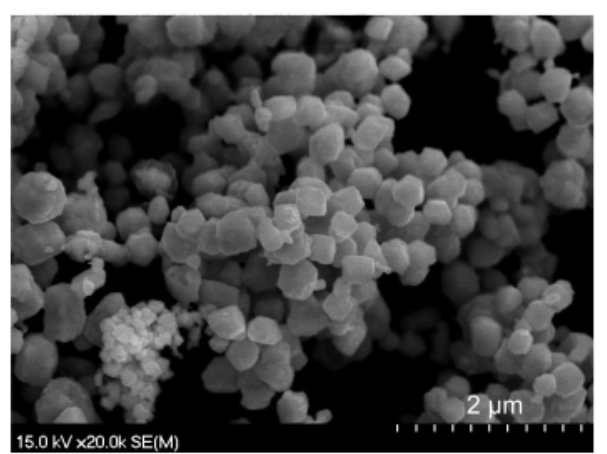

图 6 样品 2 的扫描电子显微镜照片

Fig.6 Scanning electron microscopy (SEM) image of the sample 2 

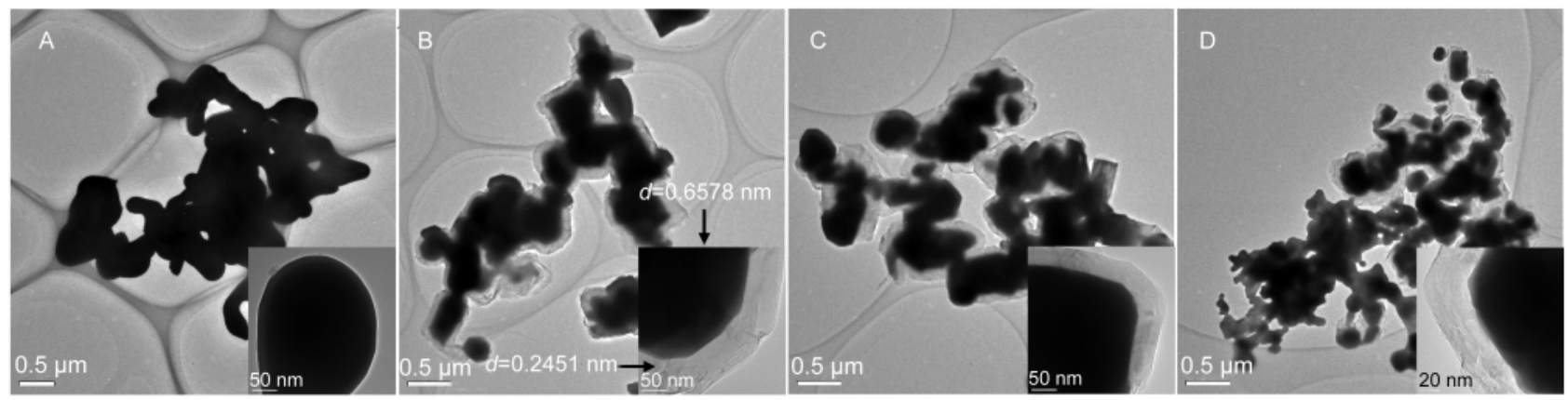

图 7 样品的 TEM 照片

Fig.7 TEM images of the samples

A: sample 1; B: sample 2; C: sample 3; D: sample 4

为了解样品颗粒的核部与壳层的晶相组成, 测 量了样品 2 中壳层和核部的晶面间距, 结果如图 7B 中所示, 其中, 壳层中有一组清晰的晶格条纹, 其间 距为 $0.2451 \mathrm{~nm}$, 这与碳化铇(PDF No. 051-0939)的 (100)晶面间距接近, 说明壳层以碳化铇为主; 核部 有一组清晰的晶格条纹, 其间距为 $0.6578 \mathrm{~nm}$, 这与 碳化铇铁(PDF No. 089-2579)的(111)晶面的晶面间 距接近, 说明核部以碳化钧铁为主. 上述结果说明 样品 2 的颗粒具有以碳化铇铁为核, 碳化铇为壳的 核壳结构. 由于样品 3 和 4 的制备方法与样品 2 相 同, 且 XRD 分析结果表明三者的晶相组成相同. 据 此可认为, 样品 $2 、 3$ 和 4 均具有以碳化钧铁为核, 碳 化铇为壳的良好核壳结构.

\subsubsection{EDS 表征}

图 8 是样品的 $\mathrm{EDS}$ 分析结果. 从图 8 中可以看 出, 样品主要含有 $\mathrm{C} 、 \mathrm{Fe} 、 \mathrm{~W}$ 和 $\mathrm{Cu}$ 元素, 其中, $\mathrm{Cu}$ 来 自测试使用的铜网, C 部分来自测试使用的碳膜, 部 分来自样品, 即样品的主要成份为 $\mathrm{C} 、 \mathrm{Fe}$ 和 $\mathrm{W}$.

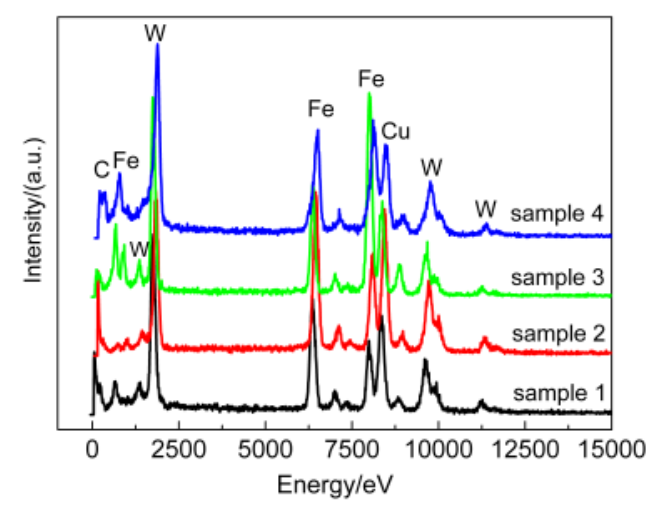

图 $8 \mathrm{AMT}$ 与 $\mathrm{FeOOH}$ 不同质量比样品的 EDS 图谱

Fig.8 Energy dispersive spectroscopy (EDS) patterns of the samples prepared with different mass ratios of AMT to FeOOH

$m(\mathrm{AMT}): m(\mathrm{FeOOH})$ : sample 1, 5; sample 2, 10; sample 3, 15; sample 4, 20
综上所述, 结合样品的 XRD、TEM 和 EDS 分析 结果, 可以推断: 样品呈典型的核壳结构, 核部主要 物相为 $\mathrm{Fe}_{3} \mathrm{~W}_{3} \mathrm{C}$, 壳层主要物相为 $\mathrm{WC}$ 和 $\mathrm{W}_{2} \mathrm{C}$.

\section{3 电化学性能}

\subsection{1 碱性体系}

图 9 是样品 $1 、 2 、 3$ 和 4 , 以及介孔空心球状碳化 铇(meso WC) 和颗粒状碳化铇(part WC) 在 $0.25 \mathrm{~mol}$ $\mathrm{L}^{-1} \mathrm{CH}_{3} \mathrm{OH}+0.1 \mathrm{~mol} \cdot \mathrm{L}^{-1} \mathrm{NaOH}$ 溶液中的循环伏安曲 线. 从图 9 中可以看到, 六个样品的扫描曲线均出现 了一个明显的氧化峰和一个还原峰. 为便于对比, 将图 9 中各样品氧化峰的峰电位和峰电流列于表 1 .

比较各样品的氧化峰电位值可知, 峰电位从低 到高依次为: 样品 $1<$ 样品 $3<$ 样品 $4<$ 样品 $2<$ meso $\mathrm{WC}<$ part WC. 与 meso WC 的氧化峰电位相比, 样品 $1 、 3 、 4$ 和 2 的氧化峰电位分别负移 $0.3200 、 0.3100$ 、 0.2867 和 $0.1749 \mathrm{~V}$. 这说明样品在碱性溶液中对 $\mathrm{CH}_{3} \mathrm{OH}$ 的电催化氧化性能均优于 meso $\mathrm{WC}$ 和 part $\mathrm{WC}$, 且样品 1 的催化性能最佳, 样品 2 的催化性能

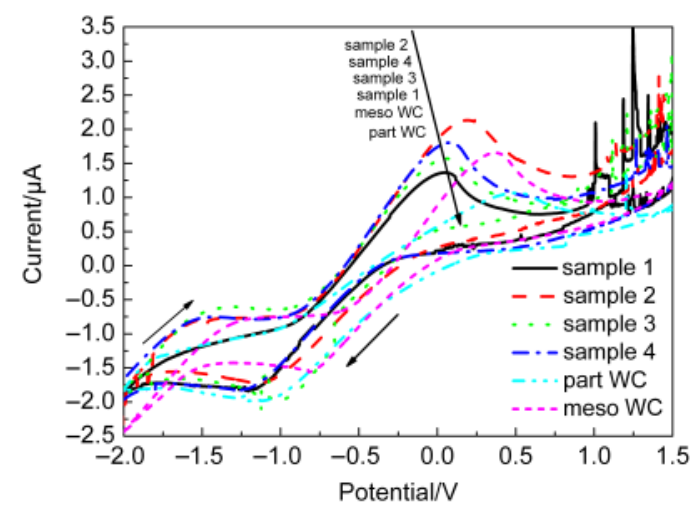

图 9 样品在 $0.25 \mathrm{~mol} \cdot \mathrm{L}^{-1} \mathrm{CH}_{3} \mathrm{OH}+0.1 \mathrm{~mol} \cdot \mathrm{L}^{-1} \mathrm{NaOH}$ 溶液 中的循环伏安曲线

Fig.9 CV curves of the samples in $0.25 \mathrm{~mol} \cdot \mathrm{L}^{-1}$ $\mathrm{CH}_{3} \mathrm{OH}+0.1 \mathrm{~mol} \cdot \mathrm{L}^{-1} \mathrm{NaOH}$ solution

scan rate: $100 \mathrm{mV} \cdot \mathrm{s}^{-1}$. meso WC: mesoporous hollow microsphere tungsten carbide; part WC: tungsten carbide particle 
表 1 图9中的氧化峰电位与及电流

Table 1 Potentials and currents from Fig.9

\begin{tabular}{ccc}
\hline \multirow{2}{*}{ Sample } & \multicolumn{2}{c}{ Oxidation } \\
\cline { 2 - 3 } & Potential/V & Current $/ \mu \mathrm{A}$ \\
\hline 1 & 0.04601 & 1.365 \\
2 & 0.1911 & 2.133 \\
3 & 0.05597 & 1.570 \\
4 & 0.07931 & 1.801 \\
meso WC & 0.3660 & 1.652 \\
part WC & 0.5004 & 1.069 \\
\hline
\end{tabular}

最差.

从表 1 中的数据可知, 电流从大到小依次为: 样 品 $2>$ 样品 $4>$ meso $\mathrm{WC}>$ 样品 $3>$ 样品 $1>$ part WC. 这 说明在碱性溶液中样品 2 的导电性最佳, 所有样品 的导电性均优于 part WC, 且样品 2 和样品 4 的导电 性还优于 meso WC.

\subsection{2 中性体系}

图 10 是样品 $1 、 2 、 3$ 和 4 , 以及 meso $\mathrm{WC}$ 和 part WC 在 $0.25 \mathrm{~mol} \cdot \mathrm{L}^{-1} \mathrm{CH}_{3} \mathrm{OH}+0.1 \mathrm{~mol} \cdot \mathrm{L}^{-1} \mathrm{NaCl}$ 溶液 中的循环伏安曲线. 从图 10 中可以看到, 六个样品 的扫描曲线均出现了一个氧化峰, 除了样品 1 外, 其 余样品的扫描曲线均出现了还原峰. 为了便于比较

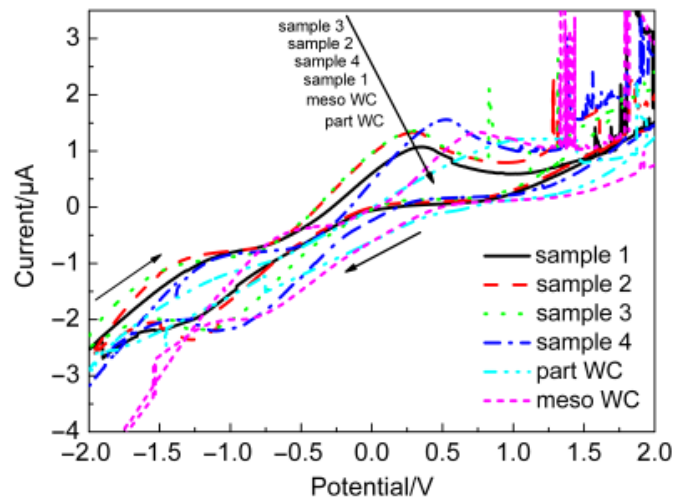

图 10 样品在 $0.25 \mathrm{~mol} \cdot \mathrm{L}^{-1} \mathrm{CH}_{3} \mathrm{OH}+0.1 \mathrm{~mol} \cdot \mathrm{L}^{-1}$ $\mathrm{NaCl}$ 溶液中的循环伏安曲线

Fig.10 CV curves of the samples in $0.25 \mathrm{~mol} \cdot \mathrm{L}^{-1}$ $\mathrm{CH}_{3} \mathrm{OH}+0.1 \mathrm{~mol} \cdot \mathrm{L}^{-1} \mathrm{NaCl}$ solution scan rate: $100 \mathrm{mV} \cdot \mathrm{s}^{-1}$

表 2 图 10 中氧化峰电位及电流

Table 2 Potentials and currents from Fig.10

\begin{tabular}{ccc}
\hline \multirow{2}{*}{ Sample } & \multicolumn{2}{c}{ Oxidation } \\
\cline { 2 - 3 } & Potential/V & Current $/ \mu \mathrm{A}$ \\
\hline 1 & 0.3534 & 1.075 \\
2 & 0.3013 & 1.339 \\
3 & 0.3043 & 1.360 \\
4 & 0.5285 & 1.558 \\
meso WC & 0.7576 & 1.338 \\
part WC & 1.106 & 1.217 \\
\hline
\end{tabular}

样品的电催化性能, 将样品的氧化峰电位和电流列 于表 2 .

从表 2 中的数据可知, 样品的氧化峰电位从低 到高依次为: 样品 $2<$ 样品 $3<$ 样品 $1<$ 样品 $4<$ meso $\mathrm{WC}<$ part WC. 与 meso WC 的氧化峰电位相比, 样品 $2 、 3 、 1$ 和 4 的氧化峰电位分别负移 $0.4563 、 0.4543$ 、 0.4042 和 $0.2291 \mathrm{~V}$. 这说明在中性溶液中, 对 $\mathrm{CH}_{3} \mathrm{OH}$ 的电催化氧化性能最佳的为样品 2 , 其次是样品 3 和 1 , 最差是样品 4 , 且均优于 meso WC 和 part WC.

比较各样品的氧化峰电流可知, 电流从大到小 依次为: 样品 $4>$ 样品 $3>$ 样品 $2>$ meso $\mathrm{WC}>$ part WC> 样品 1 . 这说明在中性溶液中, 除样品 1 外, 其余样品 的导电性均优于 meso WC 和 part WC, 导电性最佳 的是样品 4 .

\subsection{3 酸性体系}

图 11 是样品 $1 、 2 、 3$ 和 4 , 以及 meso $\mathrm{WC}$ 和 part WC 在 $0.25 \mathrm{~mol} \cdot \mathrm{L}^{-1} \mathrm{CH}_{3} \mathrm{OH}+0.1 \mathrm{~mol} \cdot \mathrm{L}^{-1} \mathrm{HCl}$ 溶液中 的循环伏安曲线. 从图 11 中可以看到, 六个样品的 扫描曲线均出现了一个氧化峰和一个还原峰. 为了 便于比较样品的电催化性能, 将样品的氧化峰电位 和电流列于表 3.

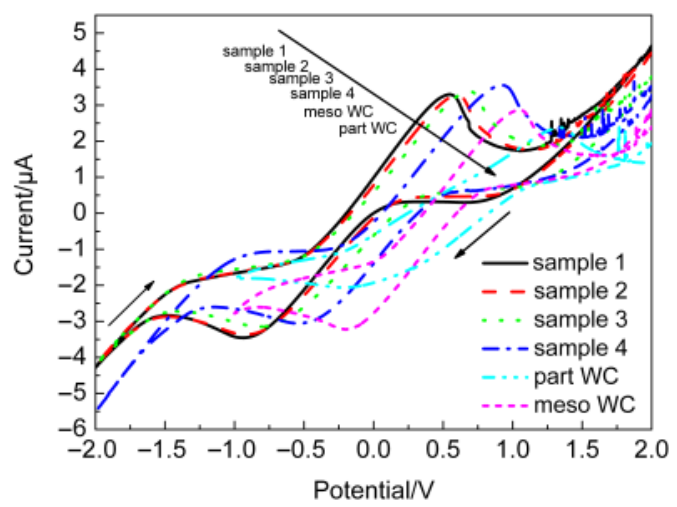

图 11 样品在 $0.25 \mathrm{~mol} \cdot \mathrm{L}^{-1} \mathrm{CH}_{3} \mathrm{OH}+0.1 \mathrm{~mol} \cdot \mathrm{L}^{-1}$ $\mathrm{HCl}$ 溶液中的循环伏安曲线

Fig.11 CV curves of the samples in $0.25 \mathrm{~mol} \cdot \mathrm{L}^{-1}$ $\mathrm{CH}_{3} \mathrm{OH}+0.1 \mathrm{~mol} \cdot \mathrm{L}^{-1} \mathrm{HCl}$ solution scan rate: $100 \mathrm{mV} \cdot \mathrm{s}^{-1}$

表 3 图 11 中的电位及电流

Table 3 Potentials and currents from Fig.11

\begin{tabular}{ccc}
\hline \multirow{2}{*}{ Sample } & \multicolumn{2}{c}{ Oxidation } \\
\cline { 2 - 3 } & Potential/V & Current $/ \mu \mathrm{A}$ \\
\hline 1 & 0.5502 & 3.297 \\
2 & 0.6008 & 3.277 \\
3 & 0.6845 & 3.379 \\
4 & 0.9248 & 3.556 \\
meso WC & 1.048 & 2.865 \\
part WC & 1.315 & 2.260 \\
\hline
\end{tabular}


从表 3 中的数据可知, 样品氧化峰电位从低到 高依次为: 样品 $1<$ 样品 $2<$ 样品 $3<$ 样品 $4<$ meso $\mathrm{WC}<$ part WC. 与 meso WC 的氧化峰电位相比, 样品 $1 、 2$ 、 3 和 4 的氧化峰电位较分别负移 $0.4978 、 0.4472$ 、 0.3635 和 $0.1232 \mathrm{~V}$. 这说明样品在酸性溶液中对 $\mathrm{CH}_{3} \mathrm{OH}$ 的电催化氧化性能均优于 meso WC 和 part WC.

比较样品的氧化峰电流值可知, 电流从大到小 依次为: 样品 $4>$ 样品 $3>$ 样品 $1>$ 样品 $2>$ meso WC> part WC. 这说明样品在酸性溶液中的导电性也优于 meso WC 和 part WC, 且导电性最佳的是样品 4 .

\section{4 分析与讨论}

\section{1 溶液性质对性能的影响}

图 12 是样品 1 在碱性、中性和酸性溶液中的循 环伏安曲线. 从图 12 中可以明显观察到, 样品 1 扫 描曲线的氧化峰电位从低到高依次为: 碱性 $<$ 中性 $<$ 酸性. 这说明样品 1 在碱性溶液中对 $\mathrm{CH}_{3} \mathrm{OH}$ 的电催 化氧化性能最佳, 其次是在中性溶液中, 最差是在 酸性溶液中.

这是因为甲醇的电催化氧化反应, ${ }^{28}$ 在酸性溶 液中的总反应式为:

$$
2 \mathrm{CH}_{3} \mathrm{OH}+3 \mathrm{O}_{2} \rightarrow 2 \mathrm{CO}_{2}+4 \mathrm{H}_{2} \mathrm{O}
$$

其中, 正极的反应为: $\mathrm{O}_{2}+4 \mathrm{e}^{-}+4 \mathrm{H}^{+} \rightarrow 2 \mathrm{H}_{2} \mathrm{O}$

负极的反应为: $\mathrm{CH}_{3} \mathrm{OH}-6 \mathrm{e}^{-}+\mathrm{H}_{2} \mathrm{O} \rightarrow 6 \mathrm{H}^{+}+\mathrm{CO}_{2}$

在碱性溶液中的总反应式为:

$$
2 \mathrm{CH}_{3} \mathrm{OH}+3 \mathrm{O}_{2}+4 \mathrm{OH}^{-} \rightarrow 2 \mathrm{CO}_{3}^{2-}+6 \mathrm{H}_{2} \mathrm{O}
$$

其中, 正极的反应为: $\mathrm{O}_{2}+4 \mathrm{e}^{-}+2 \mathrm{H}_{2} \mathrm{O} \rightarrow 4 \mathrm{OH}^{-}$ 负极的反应为: $\mathrm{CH}_{3} \mathrm{OH}-6 \mathrm{e}^{-}+8 \mathrm{OH}^{-} \rightarrow \mathrm{CO}_{3}{ }^{2-}+6 \mathrm{H}_{2} \mathrm{O}$

由此可知, 甲醇在酸性和碱性溶液中的电催化 反应有所不同, 酸性溶液中 $\mathrm{H}^{+}$主要起导电作用, 而

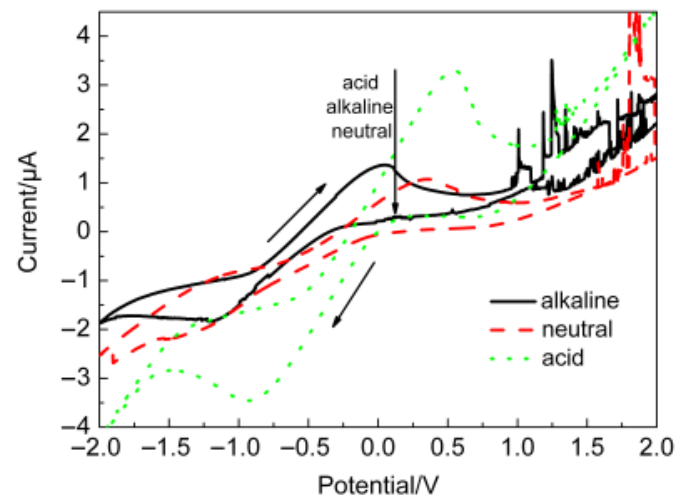

图 12 样品 1 在各种 $\mathrm{CH}_{3} \mathrm{OH}$ 溶液中的循环伏安曲线

Fig.12 CV curves of the samples in different $\mathrm{CH}_{3} \mathrm{OH}$ solutions scan rate: $100 \mathrm{mV} \cdot \mathrm{s}^{-1}$
不参与实际的反应; 碱性溶液中 $\mathrm{OH}^{-}$不仅具有导电 作用, 还参与具体的化学反应, 即反应体系中 $\mathrm{OH}^{-}$的 量越多的, 越能促进反应向右进行.

比较样品 1 在不同溶液中的导电性, 发现其在 酸性溶液中的氧化峰电流明显高于在碱性和中性 溶液中的电流. 这是与酸性溶液中 $\mathrm{H}^{+}$浓度较高且参 与导电有关.

将样品 $1 、 2 、 3$ 和 4 , 以及 meso WC 和 part WC 对 $\mathrm{CH}_{3} \mathrm{OH}$ 的电催化氧化性能进行比较发现, 在碱性溶 液中, 样品对 $\mathrm{CH}_{3} \mathrm{OH}$ 的电催化性能从强到弱依次 为: 样品 $1>$ 样品 $3>$ 样品 $4>$ 样品 2 , 且均优于 meso $\mathrm{WC}$ 和 part WC; 在中性溶液中, 样品对 $\mathrm{CH}_{3} \mathrm{OH}$ 的电 催化性能从强到弱依次为: 样品 $2>$ 样品 $3>$ 样品 $1>$ 样 品 4 , 且均优于 meso WC 和 part WC 样品; 在酸性溶 液中, 样品对 $\mathrm{CH}_{3} \mathrm{OH}$ 的电催化氧化性能从强到弱依 次为: 样品 $1>$ 样品 $2>$ 样品 $3>$ 样品 4 , 且均优于 meso $\mathrm{WC}$ 和 part $\mathrm{WC}$ 样品. 比较样品在不同性质溶液中的 氧化峰电位高低可发现, 样品在碱性溶液中的氧化 峰电位最低, 其次是在中性溶液中, 最差是在酸性 溶液中, 即样品在碱性溶液中的电催化活性最高, 其次是在中性溶液中, 最差是在酸性溶液中. 这说 明样品在碱性溶液中的催化性能均优于其在中性 和酸性溶液中的催化性能. 这是因为 $\mathrm{CH}_{3} \mathrm{OH}$ 的电催 化氧化反应须有大量 $\mathrm{OH}^{-}$的参与, 碱性 $\mathrm{CH}_{3} \mathrm{OH}$ 溶液 中有充足的 $\mathrm{OH}^{-}$, 从而促进 $\mathrm{CH}_{3} \mathrm{OH}$ 氧化反应的进 行, 致使样品在碱性溶液中的电催化氧化性能优于 其在中性和酸性溶液中的催化性能.

\section{2 物相与性能关联性}

从图 9-12 中可看出, 当电势电位高于 $1.0 \mathrm{~V}$ 时, 在不同的体系中均出现了明显的尖锐峰. 这是由于 样品中含有少量铁, 以及碳化铇铁中的铁在循环伏 安过程中能与溶液中的 $\mathrm{H}^{+}$离子发生电子交换反应, 并释放出一定量的氢气所致.

由于中性溶液中 $\mathrm{OH}^{-}$浓度与 $\mathrm{H}^{+}$的浓度处于平 衡状态, 因此, 在中性溶液中的催化性最能体现样 品的本征特性. 样品本征催化活性的强弱顺序为样 品 $2>$ 样品 $3>$ 样品 $1>$ 样品 4 . 依据 XRD 分析结果, 样品 1 中 $\mathrm{W}_{2} \mathrm{C}$ 和 $\mathrm{WC}$ 的含量最多, 其次是样品 2 和样 品 3 , 样品 4 中 $\mathrm{WC}$ 和 $\mathrm{W}_{2} \mathrm{C}$ 的含量最少. 据此可以推 断, 样品中对 $\mathrm{CH}_{3} \mathrm{OH}$ 起主要催化作用的是 $\mathrm{WC}$ 和 $\mathrm{W}_{2} \mathrm{C}$.

中性溶液中不同样品的导电性能为: 样品 $4>$ 样 品 $3>$ 样品 $2>$ 样品 1 . 结合样品的 XRD 分析结果, 四 
个样品中样品 4 的 $\mathrm{Fe}_{3} \mathrm{~W}_{3} \mathrm{C}$ 含量最高, 样品 1 的 $\mathrm{Fe}_{3} \mathrm{~W}_{3} \mathrm{C}$ 含量最低. 这说明样品中 $\mathrm{Fe}_{3} \mathrm{~W}_{3} \mathrm{C}$ 一方面作 为载体, 另一方面主要起导电作用.

综上所述, 无论在碱性、中性还是酸性溶液中, 碳化铇/碳化铇铁复合材料对 $\mathrm{CH}_{3} \mathrm{OH}$ 的电催化氧化 性能均优于 meso WC 和 part WC; 碳化铇/碳化铇铁 复合材料中在不同溶液中的催化性能具有一定的 规律性, 并与样品的物相组成有一定的相关性, 对 于 $\mathrm{CH}_{3} \mathrm{OH}$ 的电催氧化反应而言, 样品的催化活性主 要与 $\mathrm{WC}$ 和 $\mathrm{W}_{2} \mathrm{C}$ 的含量相关, 样品的导电性主要与 $\mathrm{Fe}_{3} \mathrm{~W}_{3} \mathrm{C}$ 的含量相关.

\section{5 结 论}

以偏铇酸铵为铇源, 以铁黄为铁源, 将表面包 覆与原位还原碳化技术结合可成功制备具有核壳 结构的碳化铇铁和碳化铇复合材料; 核壳结构的特 征是以碳化铇铁为核, 以碳化铇和碳化二铇为壳.

核壳结构复合材料在碱性、中性和酸性溶液中 对甲醇均具有一定的催化活性, 且均高于介孔空心 球状碳化铇和颗粒状碳化铇的电催化活性; 在上述 三种体系中, 以碱性溶液中的电催化活性最高, 其次 是中性溶液, 酸性溶液最差; 核壳结构复合材料的电 催化活性不仅与其物相组成有关, 还与其微结构特 征相关. 这些结果说明, 复合材料的电催化活性可以 通过改变反应体系的性质, 以及控制材料本身的物 相和结构特征进行调控. 上述结果充分说明核壳结 构是提高碳化铇电催化活性的有效途径之一.

\section{References}

(1) Levy, R. B.; Stauffer, M. C. Science 1973, 181, 547. doi: 10.1126/science.181.4099.547

(2) Böhm, H. Nature 1970, 227, 484.

(3) Wang, G. J.; Liu, R. Z.; Chang, J. S. J. Qingdao Univ. 2001, 16 (3), 51 [王广建, 柳荣展, 常俊石. 青岛大学学报, 2001, 16 $(3), 51$.

(4) Zhu, L. Z.; Chen, Y. F.; Zhang, Q. Y. Chin. J. Appl. Chem. 1999, 16 (4), 52. [朱龙章, 陈宇飞, 张庆元. 应用化学, 1999, 16 (4), 52.]

(5) Ma, C. A.; Yang, Z. W.; Zhou, Y. H.; Zha, Q. X. Acta Phys. -Chim. Sin. 1990, 6 (5), 622. [马淳安, 杨祖望, 周运鸿, 查全性. 物理化学学报, 1990, 6 (5), 622.] doi: 10.3866/PKU. WHXB19900521

(6) Palanker, V. S.; Gajyev, R. A.; Sokolsky, D. V. Electrochim. Acta 1977, 22, 133.

(7) Zellner, M. B.; Chen, J. G. Catal. Today 2005, 99, 299. doi: 10.1016/j.cattod.2004.10.004

(8) McIntyre, D. R.; Burstein, G. T.; Vossen, A. J. Power Sources
2002, 107, 67. doi: 10.1016/S0378-7753(01)00987-9

(9) Günter, S. E.; Detlef, B.; Walter, S. J. Catal. 1976, 43, 353. doi: 10.1016/0021-9517(76)90321-3

(10) Chen, Z. Y.; Shi, M. Q.; Ma, C. A.; Chu, Y. Q.; Zhu, A. J. Power Technology 2013, 235, 467. doi: 10.1016/j.powtec.2012.10.013

(11) Bosco, J. P.; Sasaki, K.; Sadakane, M.; Ueda, W.; Chen, J. G. Chem. Mater. 2010, 22, 966. doi: 10.1021/cm901855y

(12) Cui, X. Z.; Zhou, X. X.; Chen, H. R.; Hua, Z. L.; Wu, H. X.; He, Q. J.; Zhang, L. X.; Shi, J. L. International Journal of Hydrogen Energy 2011, 36, 10513. doi: 10.1016/j.ijhydene.2011.06.050

(13) Yan, Y.; Zhang, L.; Qi, X.; Song, H.; Wang, J. Y.; Zhang, H.; Wang, X. Small 2012, 21, 3350.

(14) Giordano, C.; Antonietti, M. Nano Today 2011, 6, 366. doi: 10.1016/j.nantod.2011.06.002

(15) Shen, P. K.; Yin, S. B.; Li, Z. H.; Chen, C. Electrochim. Acta 2010, 55, 7969. doi: 10.1016/j.electacta.2010.03.025

(16) Kumar, A.; Singh, K.; Pandy, O. P. Journal of Refractory Metals and Hard Materials 2011, 29, 555. doi: 10.1016/j. ijrmhm.2011.01.009

(17) Reddy, K. M.; Rao, T. N.; Radha, K.; Joardar, J. Journal of Alloys and Compounds 2010, 494, 404. doi: 10.1016/j. jallcom.2010.01.059

(18) Zhou, X. S.; Qiu, Y. J.; Yin, J.; Gao, S. International Journal of Hydrogen Energy 2011, 36, 7398. doi: 10.1016/j. ijhydene.2011.03.081

(19) Rahsepar, M.; Pakshir, M.; Nikolaev, P.; Safavi, A.; Palanisamy, K.; Kim, H. Applied Catalysis B: Environmental 2012, 127, 265. doi: 10.1016/j.apcatb.2012.08.032

(20) Li, G. H.; Tian, W.; Tang, J. Y.; Ma, C. A. Acta Phys. -Chim. Sin. 2007, 23 (9), 1370. [李国华, 田 伟, 汤俊艳, 马淳安. 物理化 学学报, 2007, 23 (9), 1370.] doi: 10.3866/PKU.WHXB20070912

(21) Yao, G. X.; Shi, B. B.; Li, G. H.; Zheng, Y. F. Acta Phys. -Chim. Sin. 2010, 26 (5), 1317. [姚国新, 施斌斌, 李国华, 郑遗凡. 物 理化学学报, 2010, 26 (5), 1317.] doi: 10.3866/PKU. WHXB20100337

(22) Tauster, S. J.; Fung, S. C.; Garten, R. L. J. Am. Chem. Soc. 1978, 100, 170. doi: 10.1021/ja00469a029

(23) Hu, X. C.; Chen, D.; Shi, B. B.; Li, G. H. Acta Phys. -Chim. Sin. 2011, 27 (12), 2863. [胡仙超, 陈 丹, 施斌斌, 李国华. 物理 化学学报, 2011, 27 (12), 2863.] doi: 10.3866/PKU. WHXB20112863

(24) Li, G. H.; Chen, D.; Zheng, X.; Xie, W. M.; Chen, Y. Acta Phys. -Chim. Sin. 2012, 28 (9), 2077. [李国华, 陈 丹, 郑翔, 谢伟沝, 程 媛. 物理化学学报, 2012, 28 (9), 2077.] doi: 10.3866/PKU.WHXB201206042

(25) Okamoto, H.; Kawamura, G.; Ishikawa, A.; Kudo, T. J. Electrochem. Soc. 1987, 134, 1653. doi: 10.1149/1.2100730

(26) Zhong, C. J.; Mathew, M. M. Adv. Mater. 2001, 13, 1507. doi: 10.1002/1521-4095(200110)13:19<>1.0.CO;2-S

(27) Cachet-Vivier, C.; Vivier, V.; Cha, C. S.; Nedlec, J. Y.; Yu, L. T. Electrochim. Acta 2001, 47, 181. doi: 10.1016/S0013-4686(01) 00549-7

(28) Frelink, T.; Visscher, W.; van Veen, J. A. R. J. Electroanal. Chem. 1995, 382, 65. doi: 10.1016/0022-0728(94)03648-M 